\title{
Drying of Porous Media
}

\author{
Nima Shokri ${ }^{1} \cdot$ Dani $\mathbf{O r}^{2} \cdot$ Noam Weisbrod $^{3}$. \\ Marc Prat ${ }^{4}$
}

Published online: 25 September 2015

(c) Springer Science+Business Media Dordrecht 2015

Drying of porous media is part of our daily experience, yet this common process is central to many environmental and engineering applications ranging from soil evaporation affecting hydrological water balance and climatic processes, to the drying of food and building materials, and driving plant life through transpiration. Drying rates from porous media may exhibit complex dynamics reflecting internal transport mechanisms and motion of phase change fronts that determine rates of drying and critically affect surface energy partitioning. These interactions and resulting drying dynamics present a challenge to the prediction of drying rates and interplay among mass and energy exchange even for fixed boundary conditions.

This special issue is an outcome of a symposium that was organized in the 6th International Conference of the Interpore Society held in Milwaukee, Wisconsin, USA, in 2014, that brought together researchers from various disciplines interested in drying of porous media. The contributions included in this special issue span a wide range of topics and mechanistic aspects of porous media drying and reinforcing the importance and similarity of mechanisms and parameters influencing drying processes at different settings, considering different spatial scales and representing a blend of experimental, mechanistic theoretical and numerical approaches. Although it should be clear that all aspects of drying could not be addressed, the contributions in this special issue will give the reader a wide overview of the field and will introduce several important open problems that are the subject of active research. These include the impact of second capillary effects (liquid films, liquid bridges) on drying, the coupling between internal and external transfers, the interplay between evaporation, ion transport

$\triangle$ Nima Shokri

nima.shokri@manchester.ac.uk

1 School of Chemical Engineering and Analytical Science, The University of Manchester, Manchester, UK

2 Department of Environmental Systems Science, ETH Zürich, Zürich, Switzerland

3 Zuckerberg Institute for Water Research, Blaustein Institutes for Desert Research, Ben-Gurion University of the Negev, Midreshet Ben-Gurion, Israel

4 Institut de Mécanique des Fluides de Toulouse, Allée du Professeur Camille Soula, Toulouse, France 
and precipitation-dissolution process, and the coupling between drying and poro-mechanical effects. In the following, we briefly describe the scope of each contribution.

Yiotis et al. (2015) presented a pore network model to describe the evaporation process from macroporous media considering various pore-scale mechanisms influencing the global drying behavior of a porous medium. Their model accounts for heterogeneity of the pore size distribution, effects of viscous flow through liquid films, and gravity and mass transfer both within the porous medium and through a boundary layer formed above the surface of the medium. Vorhauer et al. (2015) used experiments and pore network simulations to investigate the drying of a 2D micromodel with noncylindrical throats. Their work illustrated the importance of the presence of discrete capillary liquid rings formed during the process on the observed drying behavior. They concluded that a pore network model with inclusion of capillary ring formation is a step toward the modeling of secondary capillary effects in drying of porous media in more complex geometries such as a random packing of particles. Haghighi and Or (2015) investigated theoretically and experimentally the interactions between wavy drying surfaces and turbulent airflow above the surface to predict evaporation rates in the presence of surface roughness. Evaporation rates from wavy surfaces could be enhanced or suppressed relative to values from smooth surfaces, depending on characteristics of surface roughness (waviness) and turbulence. Jambhekar et al. (2015) investigated the evaporation process from saline porous media and presented a numerical model to describe the coupling between the free-flow region above the surface and the transport properties of porous media influencing the drying behavior. Norouzi Rad et al. (2015) used X-ray tomography to quantify the effect of grain and pore size on salt precipitation during evaporation from porous media and its consequences on the dynamics and patterns of salt precipitation in saline porous media. Nachshon and Weisbrod (2015) addressed the topic of subflorescent salt precipitation during evaporative drying and subsequent feedback on the evaporation process from saline porous media. The various impacts of efflorescent and subflorescent on evaporation rates and on modification of hydraulic characteristics of the porous medium due to salt precipitation are quantified. Scherer (2015) provides in-depth analyses of concrete drying including effects of shrinkage on compromising the resulting strength. The analysis provides new insights into the origins of the drying stress developed during drying of concrete with the associated crack formation. Shokri et al. (2015) studied formation of desiccation cracks in saline clay layers with focus on the resulting cracking patterns and dynamics as influenced by salt concentration.

The special issue provides a snapshot of the range of issues and contemporary understanding of drying porous media, and issues shared among different scientific and engineering fields. We wish to thank the many dedicated reviewers for their professional and constructive efforts to improving the quality of the contributions. We are also grateful for professional and timely assistance of the Editorial office of Transport in Porous Media, and we thank the contributing authors to this special issue.

\section{References}

Haghighi, E., Or, D.: Evaporation from wavy porous surfaces into turbulent airflows. Transp. Porous Med. (2015). doi:10.1007/s11242-015-0512-y

Jambhekar, V.A., Helmig, R., Schroder, N., Shokri, N.: Free-flow-porous-media coupling for evaporationdriven transport and precipitation of salt. Transp. Porous Med. (2015). doi:10.1007/s11242-015-0516-7

Nachshon, U., Weisbrod, N.: Beyond the salt crust: on combined evaporation and subflorescent salt precipitation in porous media. Transp. Porous Med. (2015). doi:10.1007/s11242-015-0514-9

Norouzi Rad, M., Shokri, N., Keshmiri, A., Withers, P.: Effects of grain and pore size on salt precipitation during evaporation from porous media: a pore-scale investigation. Transp. Porous Med. (2015). doi: $10.1007 / \mathrm{s} 11242-015-0515-8$ 
Scherer, G.W.: Drying, shrinkage, and cracking of cementitious materials. Transp. Porous Med. (2015). doi:10. 1007/s11242-015-0518-5

Shokri, N., Zhou, P., Keshmiri, A.: Patterns of desiccation cracks in saline bentonite layers. Transp. Porous Med. (2015). doi:10.1007/s11242-015-0521-X

Vorhauer, N., Wang, Y.J., Kharaghani, A., Tsotsas, E., Prat, M.: Drying with formation of capillary rings in a model porous medium. Transp. Porous Med. (2015). doi:10.1007/s11242-015-0538-1

Yiotis, A.G., Salin, D., Yortsos, Y.C.: Pore network modeling of drying processes in macroporous materials: effects of gravity, mass boundary layer and pore microstructure. Transp. Porous Med. (2015). doi:10. 1007/s11242-015-0529-2 